\title{
Time and temporality in library and information science
}

\author{
Jutta Haider, Veronica Johansson and Björn Hammarfelt \\ Swedish School of Library and Information Science, University of Borås, \\ Borås, Sweden
}

\begin{abstract}
Purpose - The article introduces selected theoretical approaches to time and temporality relevant to the field of library and information science, and it briefly introduces the papers gathered in this special issue. A number of issues that could potentially be followed in future research are presented.

Design/methodology/approach - The authors review a selection of theoretical and empirical approaches to the study of time that originate in or are of particular relevance to library and information science. Four main themes are identified: (1) information as object in temporal perspectives; (2) time and information as tools of power and control; (3) time in society; and (4) experiencing and practicing time.

Findings - The paper advocates a thorough engagement with how time and temporality shape notions of information more broadly. This includes, for example, paying attention to how various dimensions of the latemodern time regime of acceleration feed into the ways in which information is operationalised, how information work is commodified, and how hierarchies of information are established; paying attention to the changing temporal dynamics that networked information systems imply for our understanding of documents or of memory institutions; or how external events such as social and natural crises quickly alter modes, speed, and forms of data production and use, in areas as diverse as information practices, policy, management, representation, and organisation, amongst others.

Originality/value - By foregrounding temporal perspectives in library and information science, the authors advocate dialogue with important perspectives on time that come from other fields. Rather than just including such perspectives in library and information science, however, the authors find that the focus on information and documents that the library and information science field contributes has great potential to advance the understanding of how notions and experiences of time shape late-modern societies and individuals.
\end{abstract}

Keywords Acceleration, Data, Documents, Information, Information theory, Research agenda, Slow movement, Sociology of time, Temporality, Time

Paper type Viewpoint

\section{Introduction}

This is a text about the myriad ways in which time and information intersect, in research, in life, in society, if they can be meaningfully separated at all. The editorial introduces selected

(C) Jutta Haider, Veronica Johansson and Björn Hammarfelt. Published by Emerald Publishing Limited. This article is published under the Creative Commons Attribution (CC BY 4.0) licence. Anyone may reproduce, distribute, translate and create derivative works of this article (for both commercial and noncommercial purposes), subject to full attribution to the original publication and authors. The full terms of this licence may be seen at http://creativecommons.org/licences/by/4.0/legalcode

The authors would like to thank the participants of the event "About time: Re-thinking information through the lens of time and temporality" at the CoLIS10 conference in Ljubljana 2019, and especially the co-organisers and presenters Jenna Hartel and Alison Hicks. The authors would also like to thank colleagues at the Swedish School of Library and Information Science at the University of Borås for their valuable feedback on an early draft of this paper at a research seminar. In particular, the authors are grateful for detailed comments from Rachel Pierce, Ola Pilerot and Eva Hemmungs-Wirtén. The support and advice of the editor of the Journal of Documentation, David Bawden, has been of great help to the authors in their work on this special issue.

Additional resources: For those wanting to experience time in other ways than written text, the thematic issue is accompanied by a collaborative playlist, available on Spotify: https://tinyurl.com/ yedmvs78.

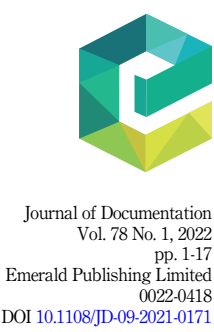


JD

78,1

2

theoretical approaches to time and temporality relevant to the field of library and information science, and it briefly introduces the papers gathered in this special issue. The complexity and richness of "time", both as a concept and as lived experience, does not lend itself to neat summaries and categorisations, and our intention is not to provide a complete overview of how time has been studied in library and information science, although we hope to cover some key contributions on the theme. Rather, in this introduction and in the special issue in general, we would like to offer a number of threads that could potentially be followed in future research. Some of these follow already established paths, while others lead into more uncharted territory. Importantly, however, all of these perspectives may inspire and enable new and fruitful questions that we believe library and information science researchers are uniquely positioned to address. Moreover, we want to draw attention to the richness of both explicit and implicit conceptualisations and theories of time and temporality that either originate from library and information science or are imported into the field.

Moving on to the topic at hand, we first note that time is one of the most fundamental structuring devices for our understanding of society. Although there are still cultural differences (see, e.g. Bluedorn, 2002), the Western originated concept of time has a global reach and dominance. It is so fundamental that we who are part of this culture cannot think without it. We do not see time, yet it helps us make sense of the world and our social lives, as various metaphors suggest. Adam (1995) highlights that time is the most commonly used noun in the English language. In English, we assert that now is the time, that the time is right, that something was bad or good timing, or that someone was on time. We lament that we do not have time, and that we are running out of time. We celebrate what is the time of our lives. We measure distances in time. Time flies by. We make time and, of course, as a society, we have learnt to accept that time is money. We talk about time all the time. But, what does that even mean? Do all these references to time refer to the same entity? Obviously, the "time" in "time is money" is different from the time we have when we are having "the time of our lives" or when "we are making time for someone". Adam (1995, p. 20) notes that "[t]he different uses of the concept entail diverse qualities and meanings of the common term. Time is multifaceted: it is involved in physical processes and social conventions, in the abstract relations of mathematics and concrete relations between people". Necessarily, then, the relations of time and information are equally multifaceted and dynamic. Philosopher Simon Critchley presents a similar argument. In a conversation with Bruno Latour, he criticises the extreme dominance of the linear notion of time, not least because it obscures the multiple ways in which time is simultaneously objective and subjective, lived and abstract (Critchley, 2016). This is similar to Adam's (1995) argument, which warns against considering different conceptions of time and time-related concepts as mutually exclusive. Rather, linear and cyclical, social and physical, biological and planetary constructs and uses of time are all, she points out, intertwined.

Conceptions of time as multiple, as exemplified above, offer a more productive means of understanding the often-difficult relationships between different concepts relating to information and time. Temporality is one such time-related term, used alongside tempo, timing or rhythm, sometimes synonymously, sometimes as an elementary condition for expressions of time and sometimes as a distinct term denoting roughly lived experiences of time. Our concern here, however, is not to develop or police a taxonomy of time-related concepts. On the contrary, we find value in how each way of employing them helps to highlight different facets of the relationships between time and information. This includes understanding how they can function both as analytical and explanatory tools and as tools for bringing together or drawing out previously unconnected time-related aspects of research objects and their relationships as units of analysis.

The issue of time is not new in studies of information, but as Savolainen (2006) notes, it has mostly been included as an implicit rather than an explicit factor. In a rare review paper in the 
field of "time and information seeking", he states that "conceptual issues of temporal factors have rarely been discussed in information studies" (Savolainen, 2006, p. 111). Overall, this statement seems justified even today. Why, then, is it interesting to think about information in relation to time? First of all, both time and information are notions with a great many colloquial, technical and academic meanings. These meanings are very powerful and have far-reaching implications. Obviously, time is not one thing, one experience or one easily defined concept. Nor is the intersection of time and information a singular point of contact. Rather, as a foundational social element, time must be seen as intricately and dynamically intertwined with the concept of information in a multitude of ways. Indeed, problems of information are frequently formulated as problems of time, and vice-versa. For example, time plays an integral part in many models of information seeking, and time constraints have been suggested as an important issue to consider in the study of information practices (for a recent example, see Bossaller et al., 2017). Moreover, information technology is implicated in the perceived acceleration of society (Moran, 2015; Wajcman and Dodd, 2016), particularly in terms of how capitalism structures progress (Nowotny, 1994; Rosa, 2015), but also in terms of how information retrieval technology and ubiquitous internet access reconfigure public and personal memories (Hellsten et al., 2006; Zavadski and Toepfl, 2019). As the reader will see, the contributions to this issue offer rich examples of versions and integrations, as well as additions to these basic notions.

The following text is divided into three main sections. The first section thematically maps a selection of theoretical and empirical approaches to the study of time that originate in or are of particular relevance to library and information science. In the subsequent section, we present and discuss the contributions to this special issue, and in the concluding section, we distill some main insights and point to potentially fruitful questions to address in further research.

\section{Theories of time and the study of information}

In what follows, we present a selection of old and new theories of time that we find represented in various ways, explicitly or implicitly, in previous research in library and information science or which we deem relevant for future work. We review these theories with a focus on their relevance to the discipline while also pointing to some empirical areas and issues that stand out as particularly significant in relation to time and information. The presentation here is organised into four themes: (1) studies of information as object in temporal perspectives; (2) time and information as tools of power and control; (3) time in society; and (4) experiencing and practicing time. This structure is also mimicked in the order of the articles in the thematic issue, as they are organised in the table of contents, according to their (main) relationships to each of these themes.

\section{Temporal aspects of information as object - data, documents, and records}

Certainly, one of the most frequently cited definitions of information within library and information science is Buckland's (1991, ch. 5) description of information as "potential evidence"; as things from which one can be informed, in short, anything that has informative potential. For the purposes of library and information science, Buckland assumes that only as material manifestations - as "recorded knowledge", "knowledge representations", "information-as-thing", described in terms of documents - can information be shared, and that this is the only form in which (technological) information systems can deal directly with information (Buckland, 1991, pp. 43, 48, 54). Thus, referring to aspects of materiality and intentionality, Buckland (as well as Levy, 2003) demarcates information in the form of documents as one of the main concerns and research objects of library and information science. This document perspective relies heavily on notions of time and temporality in several respects. 
JD

78,1

4

From the outset, the document concept points to physical manifestations of information that are characterised by permanence and stability in a temporal perspective. As described by Hjørland (2000, p. 35), it implies an entity of certain and significant stability and duration, something that "... has a history, one or more authors or producers, a connection to other documents, and so on" (c.f. also Windfeld Lund, 2010). These characteristics of documents, such as a book, a calendar, a note sheet, a receipt or a medical prescription, greatly shape the ways we as a social collective and individual users perceive, interact with and recreate information. They enable the purposeful organisation of collections of individual documents into larger units, such as archives, libraries, and museums, which in itself adds several layers of meaning and implications for everyday use and scholarly study of documents. Trusting, for example, that documents persist over time and often exist in or can be recalled or produced on-demand, as several exemplars and/or with little to no temporal restraints, sets the foundation for information practices such as reading, picking up medication or doing one's tax return. Inversely, knowing that a particular document is unique, irreplicable and irreplaceable will normally lead to vastly different approaches and practices. The perceived present or future value, persistence, and availability of a document is often rooted in historical accounts and experiences. This shapes the measures with which it is treated in the present, yet the order and relationships of these and other temporalities of documents are also frequently reversed, intermingled and iterative.

The historical roots of documents may have directed professional practice, and theoretical and empirical research approaches to aspects of permanence, long-term value, and preservation, but continuing technological development and changing social and natural conditions are opening new perspectives and raising urgent challenges. For example, although "information-as-thing" is still often represented as durable objects characterised by (physical) materiality and intentionality, such as texts, images, maps, and diagrams on paper or in digital file formats, today we also have extensive speech and face recognition, streaming data, live capture and analysis, and continuous self-learning in information systems with varying degrees of automation features and artificial intelligence ambitions (e.g. Armstrong et al., 2019; Lee and Cook, 2020). Other examples illustrate how unexpected, large-scale events dramatically change needs and practices of data and document production and use for risk assessment and response, and for resilience measures on social, organisational, and individual levels as described in relation to events such as wildfires and terrorist attacks (Borglund, 2020; Compton, 2020) and global pandemics (Harrison et al., 2021; Johansson et al., 2021; Lloyd and Hicks, 2021). The examples both require and enable rethinking of data, documents and associated information practices to account for the temporal dynamics at play.

An early example of a temporal extension of the concept of document was presented by Levy (2003). In redefining all documents, whether material in the physical sense or digital, as "representational artefacts" or "talking things", they were also explicitly understood as both fixed and fluid; simply embedded in and shaped according to social practices with varying patterns of stasis and change. Accordingly, printed books are considered to exhibit a kind of controlled, if slow, movement and change over time through annotation, reprinting, etc., whereas digital material can be stored (Levy, 2003, pp. 36-37). The latter is supported not least by the ongoing efforts to find secure and sustainable preservation solutions for the rapidly growing amounts of born-digital data and information.

Recently, therefore, the increasing production, valuation of, and dependence on data in contemporary society presents library and information science approaches to information-asobject ("information-as-thing") with particularly intriguing questions. We have both Big Data and Small Data, and their values are sometimes pitted against each other (e.g. Kitchin and Lauriault,2015), but regardless of scale: as the tools for data capture and analysis become more available, the actors involved range from supra- and international organisations, states, 
corporations, and non-governmental organisations (NGOs), to local communities and individuals, and the question of who monitors whom is not necessarily top-down, as indicated by examples in Johansson and Stenlund (this issue). We are beginning to understand how all scales - including temporal - relating to the production, organisation, analysis, and use of information as data are radically changing, but what does this mean for the notion of documents and library and information science approaches to information through this lens? When and how can "data" be considered "documents", and why, if at all, are such questions warranted and interesting from a library and information science perspective? An answer to this question and a thought-provoking contribution to the problem complex is provided by Seberger's article in this issue. Here, he introduces the notion of a "data perfect tense" to explicate shifts in conditions and consequences as individuals' interactions with ubiquitous computing engender data as "records", forming multitudes of variously enduring and interconnected, personalised, "archives" of past events, imbued in constructions of the present.

The theoretical build-up on information as object in temporal perspectives provides the basis for exploring new forms of information in terms of documents, not least the multiplicity of dynamic and data-based/data-driven information resources from personal to supranational settings. As the temporal understanding of information objects becomes richer, more complex, multifaceted and dynamic, so too do our tools for studying and understanding users' interactions, meaning-making and negotiations with these objects. The theme in the following section describes precisely such theoretical approaches, in which the material and representational qualities of documents and the users' interactions are seen as mutually shaping - and, moreover, are considered from critical perspectives.

\section{Time and information as tools for structure, control and power}

Control over time is as essential as control over space or information, and considerable power is invested in such attempts. Indeed, as the historian and philosopher Mumford (1934) points out, the invention of the mechanical clock by Benedictine monks in the 12th and 13th centuries led to one of the most fundamental technological changes in the history of the world (c.f. also Dohrn-van Rossum, 1996). Originally conceived as a means of bringing structure and order to daily life and routines in monasteries, with not least seven fixed devotional times during the day to keep track of, the more general implications of the clock in terms of synchronising and controlling collective and social action were quickly taken up and used outside these garrisons of Christianity. As abstract time (hours, minutes, seconds) replaced organic time (seasons, night, day) in regulating work and leisure, the clock, as Mumford illustrates, became more important than even the steam engine to the Industrial Revolution and its associated patterns of life. The mechanical measurement of time allowed for a whole new dimension of regulation and standardisation of working hours, production processes, and products (Dohrn-van Rossum, 1996). Later, the media theorist Neil Postman (1992, pp. 14-15) notes the irony of how the clock of the monasteries ultimately (and very quickly) came to serve mammon (capitalism) rather than the Christian God (divine devotion).

Within library and information science, information organisation concerns encompass all the tangible and intangible tools and associated practises for efficiently organising, representing, contextualising, and retrieving information and documents. In these contexts, the potential consequences of time and the various constructs associated with it from a power and control perspective are of frequent concern in numerous different application areas. Naturally, classification systems have received particular attention as reflecting and reinforcing political interests, dominant worldviews, institutionalised communication genres, and popular discourses (e.g. Bowker and Star, 2000; Olson, 2001; Tennis, 2008). Johansson (2012) extends this critical project to examine the use of temporal representations in data visualisations, highlighting how explanations for their prevalence can range from

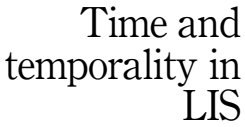


JD

78,1

unreflective conformity to organisational and diagrammatic norms and traditions, to more explicit desires to control resources and hold actors accountable. Relatedly, at the intersection of visual theory, cultural criticism and information studies, Johanna Drucker (e.g. 2014, 2020) discusses the whole of "graphesis" - the domain of visual forms of knowledge production and representation - in a call for new and alternative representations of temporality that meet the needs of the humanities, where knowledge is primarily interpretive in character, to complement the dominant logics of the natural and empirical sciences.

In an attempt to break out of the traditional, limited and limiting representations and classifications of time that pervade most information systems, Buckland et al. (2006), through their "time period directory", describe how an entire "metadata infrastructure" with particular and multifaceted focus on and representations of time can support information search and the traversal of documents beyond the previously limited constraints of datespecific search criteria and keywords. By associating the more traditional structures of information search and retrieval systems with common chronological expressions such as "Elizabethan Age", novel ways of identifying whole groups of time-related documents and information that relate to canonical periods, eras and events are supported. They write:

This work stems from a vision of future online information resources where the user can easily, and effectively, explore the historical context and interconnections of people, topics, location, and events. We expect the results of interaction with such systems to yield a rich dynamic portal of interconnected resources with maps, biographies, timelines and chronologies, in place of flat lists of web pages or bibliographic records (Buckland et al., 2006, p. 152).

Thus, as an essential feature of any classification or information system is its temporal dimension, time can thereby become an instrument of control, power and quantification (c.f. Sampson, 2020). Critical perspectives in this context mandate that with each new content, each new modality and each new user interaction, we need to question and problematise which modes of knowing, which types of knowledge, which ways of thinking, and which actors and interests that support or hinder these temporal representations, and what the implications are or might be. Consequently, these critical perspectives emphasise that the ways in which information is (temporally) represented and organised are likely to reflect prior interest or bias in ways that reconstitute and reinforce such biased ways of seeing and understanding the world and the things and relationships in it.

Expressions of temporal control, bias and oppression, however, need not be quietly and unknowingly accepted by users of the information resources that incorporate and mediate them. A vivid example of the many imaginative and empowered ways in which users identify, resist, challenge, negotiate and evade limiting and excluding temporal perspectives is provided by McKenzie (this issue) in her close analysis of users' interactions with calendars and planners in their daily lives. In a similar vein, the article by Johansson and Stenlund (this issue) presents common examples of biased temporal representations in data visualisation along with a conceptual discussion of how designers and users can engage with and challenge them through critical design and critical literacy approaches.

Taken together, the examples in this theme underscore that understanding "time" both as a feature inherent in particular representations of information and as an "external context" of social temporalities that influences how information and classification systems are designed provide another argument for why and how the study of time in relation to information is central.

Rhythms of the world - time in society

Examples of time-related expressions are sayings or proverbs that have long been part of our social vocabulary; most of the time, we do not think about them, but they are powerful; nonetheless, because they reflect normative assumptions that permeate our values, 
prioritisations, and justifications of actions and judgements. Workplaces and the market rely heavily on mechanised, rational, linear and progressive modern understandings of time and temporality, and the way information is inserted here largely reflects this. For example, social and economic development is often framed as a time and information problem, with the acceleration of access to more information presented as part of the solution to problems associated with economic progress (Haider, 2018). Typically, an allochronistic (Fabian, 1983) framing positions the poorer part as less mature and thus in a temporal distance from the richer part, resulting in rankings of development performance and economic growth. However, as exemplified in this issue by Harviainen, Lehtonen and Kock, there are settings, such as the Finnish game design community, that operate in a somewhat more cooperative manner and thus under a different temporal logic in terms of information sharing.

A similar way of explaining inequalities in terms of temporal distance, expressed as lack of information, can be seen in how a simplistic understanding of information literacy as digital skills pits generational groups against each other. Here, lifetime plays a key role in conceptualisations of information as a social and economic resource, with the young typically seen as having too much access to information (or not of the right kind) and the old too little. This reinforces a sense of urgency while evoking images of obsolescence. Both are examples of chronopolitical framing of information that, in one case, reinforces economic stereotypes and, in the other, ties into biological and social prejudices about age and technological change.

The sociology of time aims to understand how different temporalities and rhythms influence actions and our perceptions of ourselves and the world. Recent work on this theme has come to emphasise acceleration as a central aspect of late modern societies (Rosa, 2015; Wajcman, 2014; Wajcman and Dodd, 2016). A common observation in this literature is that life is getting faster; we produce and consume more, we travel faster, further and more frequently, and the experience of time pressure is epochal to (a defining feature of the latemodern experience. Sociologist Rosa (2015) identifies three interrelated but distinct types of acceleration. The first, and perhaps the easiest to identify, is technological acceleration. This is the most easily measured aspect of acceleration, with the increasing speed of transportation and information being the best examples. The second aspect is the acceleration of social change, evident, for example, in the speed with which family structures, lifestyle or other social practices and ties change. The third and final aspect is the acceleration of the pace of life itself, which amounts to an increase in the number of actions or experiences we have in a given period of time. Overall, then, what acceleration has in common in all of these cases is that it means that more units (miles, bytes, partners or experiences) are squeezed into a given period. Thus, what Rosa calls "the escalatory logic characteristic of modernity" (Rosa, 2015, p. 307) leads to an ever-increasing scarcity of time.

The detrimental effects of time pressure described by Rosa have led to calls for a general slowdown of work and life. The so-called slow movement, which first began as a protest against fast food in Italy, has since expanded to include a range of activities. Calls for deceleration are focused on the individual and on particular contexts or situations, rather than on society as a whole. Specific calls for deceleration in a particular situation (e.g. our demand for more time for ourselves and our family) can often result in others (e.g. the cashier at the local supermarket or the car in front of us) having to speed up. Examples of this phenomenon are also provided by Karen P. Nicholson (this issue) when she shows that librarians have to accelerate their work to meet the needs of others. The neoliberal university, as Nicholson $(2016,2019)$ points out, is in fact premised on a specific relationship between time and work whose fragmented and accelerating time regime has profound implications for the academic library and librarianship, from collection building to information literacy instruction (c.f. also Drabinski, 2014).

Rosa suggests, “... selective deceleration in complex and networked societies is only possible to a very limited degree.” (Rosa, 2015, p. 86). While major unforeseen events, such as 
JD

78,1

8

a pandemic, may provide opportunities for slowing down, we should be aware that even though COVID-19 has resulted in a standstill in many respects, it has also brought about a period of acceleration in many other areas: for parents juggling home-schooling and working at the same time, for those working with home delivery services, in health care and medical research, and also for those applying for benefits, if available, dealing with insurance or finding new sources of income. Thus, Rosa argues, what is called for is not a slowdown but a change in direction (Rosa, 2020). In such a reading, the "slow movement" can be seen less as a cure and more as a symptom of acceleration. Similarly, while calls for "slow" information science raise awareness of undesirable outcomes that the acceleration of late-modern life and societies might bring, we suggest that we should not merely prescribe a treatment or a counter-movement - if indeed "slow" can be seen as a solution (c.f. also Nicholsson, 2016). Rather, what is called for is a more thorough engagement with how acceleration and temporality shape notions of information more broadly. That is, we need to pay attention to how the various dimensions of the late-modern temporal regime of acceleration feed into the ways in which information is operationalised in areas as far apart as information practices, policy, management, representation, or organisation.

Theories of time and acceleration, such as those formulated by Rosa and Wajcman, among others, can help us further explore temporal dimensions of information and, conversely, help us understand informational dimensions of time and temporality. Such perspectives, and in particular, the concept of slowness, have also entered the library and information science literature when discussing personal information management (Poirer and Robinson, 2014), databases (Feinberg, 2017), and scholarly publishing (Cronin, 2013), information literacy (Nicholsson, 2016) or academic librarianship (Nicholsson, 2016). Activities that have received considerable attention in the library and information science literature, such as information overload and information avoidance, could fruitfully be seen as responses to a situation in which information is flowing faster and faster, but we still seem to be uninformed, perhaps even less well informed than before. Indeed, misinformation or disinformation seem to thrive in an ever-accelerating media landscape. Rosa's (2015) description of a "frenetic standstill", a situation in which we run faster and faster but remain at a standstill, seems to apply here as well. This contradiction appears as a key issue that information science needs to pay attention to. In particular, there is a need for research to address technological acceleration and how increasing speed and access to information, e.g. through mobile devices, shapes information practices, but also working conditions in the information professions.

One area in which acceleration and its consequences have received special attention in recent years is studies of science and scholarly communication. Studies of academics' experiences and management of time have shown that academics are increasingly pressed for time in their daily lives (Müller, 2014; Vostal, 2015). Time constraints and the increase in temporary positions contribute to a further sense of turbulence, stress and precariousness in academics' professional and personal lives (Ylijoki and Mäntylä, 2003). Thus, temporal dimensions are central to understanding the use of bibliometric measurements and their role in reinforcing a rather one-dimensional "trajectory" of academic excellence (Felt, 2017; Hammarfelt et al., 2020). Similarly, recent policy on "open science" is inextricably linked to the notion that research needs to "accelerate" to keep pace with ever-increasing global competition (Haider, 2018). The consequences of acceleration on key infrastructures of scholarly communication, book publishing, academic journals, research libraries and data repositories remain largely unexplored, although temporal perspectives and theories are central to understanding the challenges faced by these institutions and the professionals associated with them.

Overall, the sociology of time highlights questions about the sustainability of information practices in a society where individuals are likely to change jobs several times, or to migrate. The acceleration of life itself may lead us to questions about how information practices, both 
in work and leisure, evolve to accommodate a faster pace. In addition, studies in library and information science, such as Serantes and Rothbauer on reading (this issue), may provide insight into how we can find moments of resonance and connectedness in everyday life.

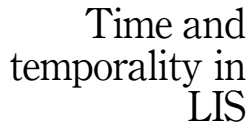

\section{Experiencing and practicing time and information}

Common ideologies and norms of time are also at work in inner, psychological conceptions of time. For example, it is not acceptable to waste someone else's time; time is precious and scarce. And for ourselves, we are supposed to be grateful for the time we are given and make the most of it, as a term like "quality time" would suggest. The situation presents the individual with the delicate dilemma of balancing radically conflicting paradigms and norms about how time should be used in a way that satisfies society's prevailing notions of what it means to use it well. It is undeniable that ideas about what kinds of information contribute to and undermine such valuable use, and how and when to engage with them, are essential to how the moral imperative is represented and experienced.

Overall, the underlying notion of time that supports conceptions of the kind described above is that of time as a stable, linear and endless continuum (see, e.g. Sampson, 2020). This understanding can be related to perceptions of time as providing stability and meaning, a sense of security and predictability - things will continue no matter what we do as individuals or as a collective. As Drucker (2014, p. 72) notes, "The idea of a celestial sphere, with its equal divisions on an ecliptic or meridian, projects a sense of rhythm, order, and regularity onto the flux of temporal change". Such perceptions are visible in psychological descriptions of time: time heals all wounds; with time, we become not only older but also wiser. On the other hand, time flies; time is cruel and relentless, time robs us of youth, health, loved ones and opportunity. Dystopian interpretations of contemporary times are prevalent in the media and culture (films, books of both social realism and science fiction character), and in such narratives, the state of the world is depicted in grim colours, with crime, violence, poverty, division, exclusion, climate change and war on the rise. Often, such narratives also portray the past with a romantic gloss.

The much-cited depiction of practices as "arrays of activities" (Schatzki, 2005, p. 2) assumes a specific temporal orientation, while the focus on routines, which is often emphasised as a defining feature, also in library and information science assumes another. The first is implicitly linear, while the other is necessarily recurrent. Thus, already by definition, the notion of practice accounts for two different temporal orientations. However, even within these two orientations, there are different timings, temporalities and rhythms. Sociologist Shove (2009, p. 25) talks of "practice-time profiles' to refer to embedded conventions of duration, sequence and timing associated with the competent performance of a practice". She also draws attention to how, through the execution of particular practices, "... recognizable infrastructures of objective time - the day, the week, the morning routine ...” come into existence (Shove, 2009, p. 19). Hartel (2010) distinguishes between different temporal arcs, each representing "a linked series of activities that unfold through time", to account for the multiple ways in which information is enacted differently depending on whether it relates to, for instance societal, personal or episodic context of a practice. Drawing on process philosophy, Savolainen (2018) elucidates the interlinking of linear and cyclical approaches in information-seeking. He suggests that these need to be seen as complementary, rather than as opposites. Tana (2019) develops what he calls an infodemiology approach to centre the role of time in health information behaviour. In particular, this foregrounds the rhythmicity of people's engagement with information and allows temporal patterns and variations in these to be explored and better understood (c.f. also Tana et al., 2019).

When time is viewed chronologically, or in terms of the measurable time of clocks and calendars (McKenzie and Davies, 2013), it forms what Savolainen (2006, p. 116) refers to as 
JD

78,1

"temporal constraints" that qualify a person's decision-making and the form and structure of their ongoing engagement with information (c.f. also Greyson, 2016). When time is understood teleologically, it can also be seen as playing a crucial role in shaping how people prepare for and are motivated to act within an information environment (Hicks, 2018). As Ronald Day demonstrates in this issue using specific situations, such meaning-making temporalities need not always be directed from the past to the present, but can also be the other way around - e.g. in therapeutic settings. Such perspectives relate to studies of information practices or behaviours and to theories of everyday life. In many ways, everyday life practices are deeply interconnected, and time is a key dimension of ordinary, everyday cultural and informational practices such as pleasure reading (Cedeira Serantes, 2016) or listening (Tattersal Wallin and Nolin, 2019), planning and organising (Davis and McKenzie, 2004), various leisure activities (Hartel, 2010), but also of how, for instance, search engines are woven into the fabric and rhythm of everyday life (Haider and Sundin, 2019).

Indeed, the notions of everyday life and practices in and of themselves are only conceivable through different conceptualisations of time (Adam, 2004; Nowotny, 1994; Shove, 2009). Everyday life, as Nowotny (1994) reminds us, is a concept rooted in a capitalist understanding of the commodification of time. Everyday life has become:

... the bracket combing work and so-called free time; the private "spending of time" and the public spending form a new combination in everyday life. The great public institutions of the state and the economy and their temporal perspectives, are confronted with the temporal perspectives of the citizens and employees, the economic subjects (Nowotny, 1994, p. 103).

She also draws our attention to the fact that the intertwining of private time and public time is increasingly happening through communication technology and, we would add, information intermediaries. This intertwining is now so profound that it has become the subject of litigation and new labour disputes. It is through information technologies - from email and general-purpose search engines to the various applications that fuel the so-called sharing or gig economy - that control over time is exercised, but private and public time also collide and collapse into one another. In this issue, this is addressed by Haider and Sundin (this issue), who propose a conceptualisation of information literacies as sites of anticipatory engagement that offer a way to make the sociomaterial conditions of information infrastructures recognisable.

\section{Eight takes on time and temporality}

As mentioned above, the purpose of this special issue and this introductory article is to present a number of possible threads that research on time and temporality in library and information science might successfully follow. The eight published articles all represent imaginative and challenging contributions that we hope will stimulate further thinking about time as a topic and theoretical perspective. The articles included not only represent a wide range of perspectives on time, but they also highlight the breadth of library and information science as a field of research. Topics such as reading, academic libraries, information sharing, ubiquitous computing, psychoanalytic notions of information, data visualisation, and information literacy are all represented. Moreover, the contributions move on different analytical levels, ranging from sociological accounts to organisational perspectives to studies of everyday practices and individual experiences. What all of these contributions have in common, however, is an effort to engage conceptually and theoretically with the complex notion of time. The included articles are here briefly introduced in order that generally follows the thematic structure outlined above.

John S. Seberger's paper, Into the archive of ubiquitous computing: the data perfect tense and the historicization of the present, argues that "ubiquitous computing" involves an ongoing 
historicisation of the present through various devices such as smartphones, tracking devices, and smart objects. This development is accelerated by increasing networking, connectivity and by economic motives in surveillance capitalism. Using the example of Sal - a fictional character who projected what a world of ubiquitous computing would look like in 1991 Seberger shows how the "now" and the "then" are merging as lives are automatically and instantly archived. At the same time, "[e]very moment of use, every automatic collection of data by a smart object, becomes a moment of evaluation and capture" (Seberger, this issue) Thus, users become familiar with descriptions of themselves and turn into objects of the archive. Understanding this fundamental shift towards a "data perfect tense", Seberger argues, is important when addressing the disjuncture between users and the devices, apps, and algorithms that surround them. Critically evaluating this temporal shift is becoming a key challenge for library and information science and related research fields.

The planning of time and information infrastructures used to do so, such as planners, calendars and spreadsheets, is the subject of Pamela McKenzie and Elisabeth Davies' (this issue) paper, Documenting multiple temporalities. Their paper shows how individuals handle "multiple temporalities" - e.g. body time, clock time and biographical time - in their everyday lives. For example, a calendar studied by McKenzie and Davies includes ideas about the menstrual cycle (bodily time), work meetings (clock time) and reminders of "falling leaves" (nature time). Nevertheless, the artefacts used for scheduling, such as calendars, are designed according to specific "temporal logics", making it easier to schedule certain types of events (e.g. work meetings) compared to others (e.g. care giving). When participants found that planning tools did not meet their demands, they often modified or invented their own devices that were better suited to their specific needs. Accordingly, the temporal logic inherent in planners and calendars shaped how time was managed, but did not fully determine how participants scheduled their lives. The paper persuasively elucidates how documentary planning tools can shed light on daily practices and bring out their "multifaceted" and "polychronic" character.

The paper by Veronica Johansson and Jörgen Stenlund entitled Making time/breaking time: critical literacy and politics of time in data visualisation addresses the challenges that temporal representations pose for data visualisations. Representations of time are often used to construct narratives in data visualisations. However, time is a value-laden concept, and representations can never provide complete, objective accounts of "temporal reality". By engaging with a selection of fascinating examples, Johansson and Stenlund explore expressions of temporal bias and politics in data visualisations. In particular, they draw attention to four different types and effects of temporal bias, namely, limitation of view, disregard of variation, oppression of social groups, and misrepresentation of topic. They propose an original way to connect critical literacy, radical literacy, and critical design, and the resulting theoretical framework promotes a sociotechnical view of representations as biased and political. By suggesting that appropriate critical and radical literacy approaches require users and designers to critique, contextualise, counter, and transcend expressions of bias, the authors make a novel and innovative contribution to understanding and problematising the chronopolitics of visual information and foreground the shared rather than conflicting interests of empirical science and the humanities.

The article Timeliness in information sharing within creative industries. Case: Finnish game design by Tuomas Harviainen, Miika Lehtonen and Sören Kock shows how specific temporal aspects of information sharing are central to the organisation of work among Finnish game designers. As Harviainen, Lehtonen and Kock argue, timeliness in information gathering and market analysis is pivotal for the success of games; otherwise well-designed products can suffer if they do not reach the market at the right moment. While game design is generally a very secretive business, the Finnish scene is surprisingly open, and information about new ideas and products is often shared between companies that are both competitors and collaborators. Information sharing takes place at meetings and parties, and the proactive 
JD

78,1

exchange serves both to enhance potential future use and to strengthen the sense of community. Indeed, information sharing is generally seen as a collaborative strength rather than a threat to individual companies. In fact, the success of game developers could be attributed to a strong community where information is shared freely and quickly. As Harviainen et al. (this issue) note, "[a]s long as the community stays tight and open in its information culture, this time-bound and timely, yet in many ways also time-free information sharing becomes a form of forward planning for the entire scene and makes it much stronger". In summary, this paper shows how temporality can be successfully used as an analytical framing to illuminate information practices in particular organisations and communities.

Karen Nicholson's conceptual analysis, entitled Spatial thinking and immaterial affective labour in the post-Fordist academic library, offers a critical discussion of how time and spatiality shape library work. Through the concept of "spatial thinking" (Soja, 2010), Nicholson rejects the dualism "... that associates time with progress, civilization, science, politics, and reason on the one hand, and space with statis, reproduction, nostalgia, aesthetics, and the body on the other" (Nicholson, this issue). The account resonates with the sociological perspective on time where the acceleration of life and work is related to the rise of neoliberalism. These developments are also discussed in relation to a feminist critique in which the emotional and caring work of academic librarians is devalued, and in which librarians need to "recalibrate" according to the temporal order of faculty and students. According to Nicholson, the accelerated pace of the academic library hinders information sharing, it disciplines library workers and it limits the possibility of inventive and critical librarianship. Overall, Nicholson convincingly demonstrates how spatio-temporal perspectives opens for critical and important insights regarding work and life in libraries.

In Reading time: exploring the temporal experiences of reading Lucia Cedeira Serantes and Paulette Rothbauer show how theories of time and temporality can be used to analyse reading practices. In doing so, they identify three distinct temporalities: the spatial time of reading, temporalised reading bodies, and an interrupted chrononormative time. A notable temporal feature of reading in their analysis is its ability to fit “... into daily routines and rhythms, but at the same time, it can be a revolt against temporal regimes. Reading in this register is about escaping time or resisting the pressure of clock time - it is about making time" (Serantes and Rothbauer, this issue). The temporality of reading, they argue, could thus be discussed as an example of what Rosa describes as "moments of resonance" in which we feel connected to and immersed in the world. It may also, as one reader of comics puts it, allow us to control time, as the reader determines the pace at which a particular page is read. As observed by Serantes and Rothbauer, the material expression of the bookshelf also situates the reader in time and space, and they leave traces of past reading experiences. Therefore, focusing on temporal aspects might open up the possibility of studying the "messiness" of reading and go beyond the usual focus on institutional or clock time.

In Information literacy as a site for anticipation: temporal tactics for infrastructural meaning-making and algo-rhythm awareness, Jutta Haider and Olof Sundin (this issue) present the results of an analysis of pair interviews with teenagers in Sweden and Denmark concerning their apprehensions of and interactions with algorithm-based information intermediaries such as search engines, streaming media, recommender services and social media. Although the participants rarely acknowledge their actions as engagements with algorithms per se, they were found to respond to, negotiate with, and challenge and even "tame" for personal utility ("gaming the algorithms") the algorithmic functions of these systems in many ways. Three main and (mostly) forward-oriented themes in terms of anticipating personalisation, divergences, and interventions extend on previous conceptions of both information literacies and intermediaries as sociomaterial constructs. Temporal factors relating to timing (rhythm), purpose of activity, and personal needs provide shifting conditions for the co-construction of information literacies in an algorithmic culture. In 
describing information literacies as sites of anticipation, the authors present a conceptual extension of the concept that adds a vital, temporally framed, point of engagement to existing critically informed information literacies theorising, research and education.

Finally, the experience of time and its relationship to information acquisition in the context of trauma is the subject of Ronald Day's study Trauma, time, and information. Trauma can generally be understood as the result of a violent event that causes damage to a normally integrated psychological self. It is often seen as having its origins in the past while affecting the future. However, trauma theory also involves another "retroactive" notion of time, called "Nachträglichkeit" in which past events are compounded by present events. Building on Freud and his thoughts on trauma, as well as Wittgenstein's critique of psychoanalysis, Day outlines in fascinating detail how representation and narration are central to understanding how information (and "facts") can be understood in relation to trauma. This also explains the close relationship with literature, as psychoanalysis "... models itself in time and observed in time as embedded in narrative devices and their ordinary causal-temporal assumptions" (Day, this issue). Therefore, "information" in trauma therapy is psychologically valuable, whether or not the information is empirically valid. However, as Day points out, expanding this understanding of useful information in psychological explanations to other contexts may be dangerous and could in the public and political sphere result in historical revisionism.

\section{Concluding remarks: towards a research agenda?}

In conclusion, our intention with compiling a selection of contemporary library and information science research that presents different approaches to exploring time and information, is also to provide a vantage point from which to identify shared concerns and key themes that unite otherwise disparate approaches. By foregrounding temporal perspectives in library and information science, we advocate dialogue with important perspectives on time that come from other fields. Rather than just including such perspectives in library and information science, however, we find that the focus on information and documents that our field contributes has great potential to advance the understanding of how notions and experiences of time shape late-modern societies and individuals.

What is called for is a thorough engagement with how time and temporality shape notions of information more broadly and, conversely, with how notions of time and temporality shape and produce objects of study for library and information science. This includes, for example, paying attention to how various dimensions of the late-modern time regime of acceleration feed into the ways in which information is operationalised, how information work is commodified, and how hierarchies of information are established; paying attention to the changing temporal dynamics that networked information systems imply for our understanding of documents or of memory institutions; or how external events such as social and natural crises quickly alter modes, speed, and forms of data production and use. The issues involved strike a chord that runs through all aspects of the discipline, involving areas as diverse as information practices, policy, management, representation, and organisation, to name just a few. It is our hope that this joint publication will itself stimulate and facilitate cross-sectional enrichment and new ideas for theoretical and empirical development concerning time and information.

\section{Articles in this issue}

Day, R.E. (this issue), "Trauma, time and information", Journal of Documentation. https://doi.org/10. 1108/JD-11-2020-0189.

Haider, J. and Sundin, O. (this issue), "Information literacy as a site for anticipation: temporal tactics for infrastructural meaning-making and algo-rhythm awareness", Journal of Documentation. https://doi.org/10.1108/JD-11-2020-0204. 
JD

78,1

Harviainen, J.T., Lehtonen, M.J. and Kock, S. (this issue), "Timeliness in information sharing within creative industries. Case: Finnish game design", Journal of Documentation. https://oi.org/10. 1108/JD-12-2020-0207.

Johansson, V. and Stenlund, J. (this issue), "Making time/breaking time: critical literacy and politics of time in data visualisation", Journal of Documentation. https://doi.org/10.1108/JD-12-2020-0210.

McKenzie, P.J. and Davies, E. (this issue), "Documenting multiple temporalities", Journal of Documentation. https://doi.org/10.1108/JD-11-2020-0196.

Nicholson, K.P. (this issue), "Spatial thinking, gender and immaterial affective labour in the postFordist academic library", Journal of Documentation. https://doi.org/10.1108/JD-11-2020-0194.

Rothbauer, P.M. and Cedeira Serantes, L. (this issue), "Reading time: exploring the temporal experiences of reading", Journal of Documentation. https://doi.org/10.1108/JD-11-2020-0200.

Seberger, J.S. (this issue), "Into the archive of ubiquitous computing: the data perfect tense and the historicization of the present", Journal of Documentation. https://doi.org/10.1108/JD-11-2020-0195.

\section{References}

Adam, B. (1995), Timewatch. The Social Analysis of Time, Polity Press, Cambridge, MA.

Adam, B. (2004), Time, Polity Press, Cambridge, MA.

Armstrong, M.P., Wang, S. and Zhang, Z. (2019), "The Internet of Things and fast data streams: prospects for geospatial data science in emerging information ecosystems", Cartography and Geographic Information Science, Vol. 46 No. 1, pp. 39-56, doi: 10.1080/ 15230406.2018.1503973.

Bluedorn, A.C. (2002), The Human Organization of Time: Temporal Realities and Experience, Stanford Univ Press, Stanford, CA.

Borglund, E.A.M. (2020), in Sundqvist, A., Berget, G., Nolin, J. and Skjerdingstad, K. (Eds), "Recordsmaking during crisis management - rule based or discretion driven?", Sustainable Digital Communities. iConference 2020. Lecture Notes in Computer Science, Vol. 12051, Springer, Cham, pp. 599-615, doi: 10.1007/978-3-030-43687-2_50.

Bossaller, J., Bossaller, J., Burns, C.S., Burns, C.S., VanScoy, A. and VanScoy, A. (2017), "Re-conceiving time in reference and information services work: a qualitative secondary analysis", Journal of Documentation, Vol. 73 No. 1, pp. 2-17.

Bowker, G.C. and Star, S.L. (2000), Sorting Things Out: Classification and its Consequences, MIT press, Cambridge, MA.

Buckland, M.K. (1991), Information and Information Systems. New Directions in Information Management, Greenwood, New York.

Buckland, M., Larson, R.R. and Petras, V. (2006), "Time period directories: a metadata infrastructure for placing events in temporal and geographic context", Proceedings of the 6th ACM/IEEE-CS Joint Conference on Digital Libraries (JCDL 'O6), Chapel Hill, NC, pp. 151-160, doi: 10.1145/ 1141753.1141782.

Cedeira Serantes, L. (2016), "When comics set the pace: the experience of time and the reading of comics”, in McKechnie, L., Rothbauer, P., Oterholm, K. and Skjerdingstad, K.I. (Eds), Plotting the Reading Experience: Theory, Practice, Politics, Ontario Wilfrid Laurier University Press, Waterloo, pp. 217-232.

Compton, C. (2020), "The temporality of disaster: data, the emergency, and climate change", (Forthcoming [2020] Anthropocenes, UNSW Law Research Paper No. 20-43, available at: SSRN: https://ssrn.com/abstract $=3677690$.

Critchley, S. (2016), “Times”, Parse, 4, available at: http://parsejournal.com/article/times/.

Cronin, B. (2013), "Slow food for thought", Journal of the American Society for Information Science and Technology, Vol. 64 No. 1, p. 1. 
Davies, E. and McKenzie, P.J. (2004), "Preparing for opening night: temporal boundary objects in textually-mediated professional practice", Information Research, Vol. 10 No. 1.

Dohrn-van Rossum, G. (1996), History of the Hour: Clocks and Modern Temporal Orders, Univ. of Chicago Press, Chicago.

Drabinski, E. (2014), "Toward a kairos of library instruction”, Journal of Academic Librarianship, Vol. 40 No. 5, pp. 480-485, doi: 10.1016/j.acalib.2014.06.002.

Drucker, J. (2014), Graphesis: Visual Forms of Knowledge Production, Harvard University Press, Cambridge, MA.

Drucker, J. (2020), Visualization and Interpretation: Humanistic Approaches to Display, The MIT Press, Cambridge, MA.

Fabian, J. (1983), Time and the Other: How Anthropology Makes its Object, Columbia University Press, New York.

Feinberg, M. (2017), "Reading databases: slow information interactions beyond the retrieval paradigm", Journal of Documentation, Vol. 73 No. 2, pp. 336-356.

Felt, U. (2017), "Under the shadow of time: where indicators and academic values meet", Engaging Science, Technology, and Society, Vol. 3, pp. 53-63.

Greyson, D. (2016), "Evolution of information practices over time", Proceedings of the Association for Information Science and Technology, Vol. 53 No. 1, pp. 1-8.

Haider, J. (2018), "Openness as tool for acceleration and measurement: reflections on problem representations underpinning open access and open science", in Schöpfel, J. and Herb, U. (Eds), Open Divide? Critical Studies on Open Access, Library Juice Press, Sacramento, CA, pp. 17-28.

Haider, J. and Sundin, O. (2019), Invisible Search and Online Search Engines: the Ubiquity of Search in Everyday Life, Routledge, London.

Hammarfelt, B., Rushforth, A.D. and de Rijcke, S. (2020), "Temporality in academic evaluation: 'trajectoral thinking' in the assessment of biomedical researchers", Valuation Studies, Vol. 7 No. 1, pp. 33-63.

Harrison, M., Lancaster, K. and Rhodes, T. (2021), “A matter of time': evidence-making temporalities of vaccine development in the COVID-19 media landscape", Time and Society, first published July 2021. doi: 10.1177/0961463X211032201.

Hartel, J. (2010), "Time as a framework for information science: insights from the hobby of gourmet cooking", Information Research, Vol. 15 No. 4, paper 19.

Hellsten, I., Leydesdorff, L. and Wouters, P. (2006), "Multiple presents: how search engines rewrite the past", New Media and Society, Vol. 8 No. 6, pp. 901-924.

Hicks, A. (2018), "The theory of mitigating risk: information literacy and language-learning in transition", The Swedish School of Library and Information Science, The University of Borås.

Hjørland, B. (2000), "Documents, memory institutions and information science", Journal of Documentation, Vol. 56, pp. 27-41.

Johansson, V. (2012), “A time and place for everything? Social visualisation tools and critical literacies", The Swedish School of Library and Information Science, The University of Borås.

Johansson, V., Wänström, L., Ramme, K., Kallio, S. and Yousefi Mojir, K. (2021), "Crowdsourcing long COVID Sweden (CiLC-S): exploring digital methods and voluntary health data for research and response in social crises", Paper Presented at the Information Science Trends The ASIS and T European Chapter Research Series, June 09-11, 2021, doi: 10.5281/zenodo. 4902175.

Kitchin, R. and Lauriault, T.P. (2015), "Small data in the era of big data”, GeoJournal, Vol. 80, pp. 463-475.

Lee, A.J. and Cook, P.S. (2020), "The myth of the 'data-driven' society: exploring the interactions of data interfaces, circulations, and abstractions”, Sociology Compass, Vol. 14 No. 1, e12749. 
JD

78,1

Levy, D.M. (2003), "Documents and libraries: a sociotechnical perspective", in Bishop, A.P., Van House, N.A. and Buttenfield, B.P. (Eds), Digital Library Use: Social Practice in Design and Evaluation, Digital Libraries and Electronic Publishing, Cambridge, MA, pp. 25-42.

Lloyd, A. and Hicks, A. (2021), "Contextualising risk: the unfolding information work and practices of people during the COVID-19 pandemic", Journal of Documentation, Vol. 77 No. 5, pp. 1052-1072.

McKenzie, P.J. and Davies, E. (2013), "Time is of the essence: social theory of time and its implications for LIS research", Proceedings of the Annual Conference of CAIS/Actes du congrès annuel de l'ACSI.

Moran, C. (2015), "Time as a social practice”, Time and Society, Vol. 24 No. 3, pp. 283-303.

Müller, R. (2014), "Racing for what? Anticipation and acceleration in the work and career practices of academic life science postdocs", Forum Qualitative Sozialforschung/Forum: Qualitative Social Research, Vol. 15 No. 3.

Mumford, L. (1934), Technics and Civilization, HarcourtBrace and company, New York.

Nicholson, K.P. (2016), “'Taking back' information literacy: time and the one-shot in the neoliberal university", in Pagowsky, N. and McElroy, K. (Eds), Critical Library Pedagogy Handbook, ACRL, Chicago, Vol. 1, pp. 25-39.

Nicholson, K.P. (2019), "On the space/time of information literacy, higher education, and the global knowledge economy", in Cifor, M. and Lee, J.A. (Eds), Evidences, Implications, and Critical Interrogations of Neoliberalism in Information Studies, Special issue, Journal of Critical Library and Information Studies, Vol. 2 No. 1, doi: 10.24242/jclis.v2i1.86.

Nowotny, H. (1994), Time: the Modern and Postmodern Experience, Polity Press, Cambridge.

Olson, H.A. (2001), "The power to name: representation in library catalogs", Signs, Vol. 26 No. 3, pp. 639-668.

Poirier, L. and Robinson, L. (2014), "Informational balance: slow principles in the theory and practice of information behavior", Journal of Documentation, Vol. 70 No. 4, pp. 687-707.

Postman, N. (1992), Technopoly: the Surrender of Culture to Technology, Knopf, New York.

Rosa, H. (2015), Social Acceleration: A New Theory of Modernity, Columbia University Press, New York.

Rosa, H. (2020), We Can Quit the Rat Race. The Sociologist Prof. Dr Hartmut Rosa Analyses the Impact of the Corona Lockdown on Individuals and the Society, interviewed by Ute Schönfelder, Friedrich Schiller Universität Jena, available at: https://www.uni-jena.de/en/200403_Rosa_ Interview (accessed 13 September 2020).

Sampson, K. (2020), "Conceptions of temporality: reconsidering time in an age of impending emergency", Theoria, Vol. 86, pp. 769-782.

Savolainen, R. (2006), "Time as a context of information seeking", Library and Information Science Research, Vol. 28 No. 1, pp. 110-127.

Savolainen, R. (2018), "Information-seeking processes as temporal developments: comparison of stagebased and cyclic approaches", Journal of the Association for Information Science and Technology, Vol. 69 No. 6, pp. 787-797.

Schatzki, T.R. (2005), "Introduction: practice theory", in Knorr Cetina, K., Schatzki, T.R. and von Savigny, E. (Eds), The Practice Turn in Contemporary Theory, Routledge, London and New York, pp. 1-14.

Shove, E. (2009), "Everyday practice and the production and consumption of time", in Shove, E., Trentman, F. and Wilk, R. (Eds), Time, Consumption and Everyday Life: Practice, Materiality and Culture, Berg, Oxford and New York, pp. 17-35.

Soja, E.W. (2010), Seeking Spatial Justice, University of Minnesota Press, Minneapolis, MN.

Tana, J. (2019), Infodemiology: Studying Rhythmicity in Online Health Information Behavior, Åbo Akademi University, Abo. 
Tana, J., Eirola, E. and Eriksson-Backa, K. (2019), "Rhythmicity of health information behaviour: utilizing the infodemiology approach to study temporal patterns and variations", Aslib Journal of Information Management, Vol. 71 No. 6, pp. 773-788.

Tattersall Wallin, E. and Nolin, J. (2020), "Time to read: exploring the timespaces of subscriptionbased audiobooks", New Media and Society, Vol. 22 No. 3, pp. 470-488.

Tennis, J.T. (2008), "Epistemology, theory and methodology in knowledge organization: toward a classification, metatheory, and research framework", Knowledge Organization, Vol. 35 Nos 2/3, pp. 102-112.

Vostal, F. (2015), "Academic life in the fast lane: the experience of time and speed in British academia", Time and Society, Vol. 24 No. 1, pp. 71-95.

Wajcman, J. (2014), Pressed for Time: the Acceleration of Life in Digital Capitalism, University of Chicago Press, Chicago.

Wajcman, J. and Dodd, N. (Eds) (2016), The Sociology of Speed: Digital, Organizational, and Social Temporalities, Oxford University Press, Oxford.

Windfeld Lund, N. (2010), "Document, text and medium: concepts, theories and disciplines”, Journal of Documentation, Vol. 66 No. 5, pp. 734-749.

Ylijoki, O.H. and Mäntylä, H. (2003), "Conflicting time perspectives in academic work", Time and Society, Vol. 12 No. 1, pp. 55-78.

Zavadski, A. and Toepfl, F. (2019), "Querying the Internet as a mnemonic practice: how search engines mediate four types of past events in Russia", Media, Culture and Society, Vol. 41 No. 1, pp. 21-37.

\section{Corresponding author}

Jutta Haider can be contacted at: jutta.haider@hb.se

For instructions on how to order reprints of this article, please visit our website:

www.emeraldgrouppublishing.com/licensing/reprints.htm

Or contact us for further details: permissions@emeraldinsight.com 